\title{
Uso de un blog docente para el desarrollo del pensamiento crítico y la capacidad de expresión oral y escrita en ciencias del deporte
}

Vicente J. Beltrán-Carrilloa, Javier Valenciano-Valcárcel ${ }^{\mathrm{b}}$ y Joan Pere MolinaAlventosa $^{\mathbf{c}}$

${ }^{a}$ Centro de Investigación del Deporte. Universidad Miguel Hernández de Elche. vbeltran@umh.es, bFacultad de Educación de Toledo. Universidad de Castillla-La Mancha. Javier.Valenciano@uclm.es

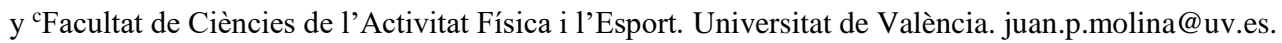

\begin{abstract}
The innovative teaching project described in this document was carried out in the subject Theory of Physical Education and Sport, belonging to the Physical Activity and Sport Sciences Degree (Miguel Hernández University of Elche). The project consisted in the use of a teaching blog to foster critical thinking and the ability of oral and written expression. The participation in the blog was related to a voluntary task which let the students increase their final mark in the subject. The students made oral comments (YouTube videos) or written comments to the entrances proposed by the lecturer. These entrances were about current topics related to physical activity and sport. At the end of the subject, the lecturer sent a Google form to the students to know their opinion about this innovative teaching project. The students' opinion regarding the utility of the blog as a teaching resource was positive.
\end{abstract}

Keywords: Blog, entrance, comment, video, reviewer, award.

\begin{abstract}
Resumen
El proyecto de innovación docente que se describe en este documento se desarrolló en la asignatura de Teoría de la Educación Física y el Deporte, perteneciente al Grado en Ciencias de la Actividad Física y el Deporte de la Universidad Miguel Hernández de Elche. El proyecto consistió en el uso de un blog docente para el desarrollo del pensamiento crítico y la capacidad de expresión oral y escrita. La participación en el blog estaba vinculada a un trabajo opcional con el que los estudiantes podían incrementar la nota final de la asignatura. Los alumnos realizaron comentarios orales (vídeos de YouTube) o escritos a las entradas propuestas por el profesor, sobre temas de actualidad relacionados con la actividad física y el deporte. Al finalizar la asignatura, el profesor envío un formulario de Google a los alumnos para conocer su opinión sobre este proyecto de innovación. La opinión de los alumnos sobre la utilidad del blog como recurso docente fue positiva.
\end{abstract}

Palabras clave: Blog, entrada, comentario, vídeo, evaluador, premio. 
Uso de un blog docente para el desarrollo del pensamiento crítico y la capacidad de expresión oral y escrita en ciencias del deporte.

\section{Introducción}

El proyecto de innovación docente que se describe en este documento se desarrolló en la asignatura de Teoría de la Educación Física y el Deporte, perteneciente al Grado en Ciencias de la Actividad Física y el Deporte de la Universidad Miguel Hernández de Elche. En esta asignatura es de 6 créditos ECTS, pertenece al primer curso de grado y se imparte durante el primer semestre. En el curso 2016-2017 se matricularon en esta asignatura 149 estudiantes.

Este proyecto docente se llevó a cabo para fomentar el pensamiento crítico entre el alumnado. El pensamiento crítico se relaciona con conocimientos y competencias que deberían potenciarse en todos los niveles educativos (Boisvert, 2004; Guzman Silva y Sánchez Escobedo, 2006; Rojas Viteri, Pérez Narváez y Álvarez Zurita, 2016). Sin embargo, muchos profesores pensamos que el pensamiento crítico no se incentiva suficientemente en las aulas universitarias, mientras que el conocimiento técnico recibe mucha más atención y se aborda en profundidad.

Junto al pensamiento crítico, la capacidad de expresión oral y escrita representa otra competencia de especial relevancia para la vida personal y profesional de los estudiantes universitarios (Peña González, 2011; Ruiz Muñoz, 2012). De manera preocupante, observamos que muchos alumnos no poseen una adecuada capacidad de expresión oral y escrita, con la que debería contar todo titulado universitario.

Por último, sabemos que, en la actualidad, los estudiantes universitarios se sienten especialmente atraídos por las nuevas tecnologías de la información y la comunicación y por las posibilidades que ofrece internet para comunicar y recibir opiniones e ideas. Concretamente, el uso de blogs docentes ha resultado ser una estrategia interesante para la innovación y la mejora de los procesos de enseñanza-aprendizaje en educación superior (Molina Alventosa, Valenciano Valcárcel y Valencia Peris, 2015; Molina Alventosa, Valencia Peris y Suárez Guerrero, 2016).

Con la intención de paliar las debilidades y aprovechar las oportunidades identificadas anteriormente, se planteó un proyecto docente cuyos objetivos se concretan en el siguiente apartado.

\section{Objetivos}

Los objetivos de este proyecto de innovación docente fueron fomentar entre el alumnado el pensamiento crítico y favorecer su capacidad de expresión oral y escrita. Estos objetivos, que están totalmente relacionados con los propósitos fundamentales de la asignatura, se abordaron mediante el uso de un blog docente al que se puede acceder en el siguiente enlace: http://teoriaefd.blogspot.com.es/

Al tratarse de un blog vivo, a comienzo del curso 2017-2018, las entradas a las que se hace alusión en este documento se eliminarán para ir introduciendo las entradas correspondientes al próximo curso.

(cc) EY-NC-ND 2017, Universitat Politècnica de València 


\section{Desarrollo de la innovación}

\subsection{El blog dentro del sistema de evaluación de la asignatura}

La participación en el blog docente estaba vinculada a un trabajo opcional con el que los estudiantes podían incrementar la nota final de la asignatura hasta 0,5 puntos, siempre y cuando aprobasen el examen de la asignatura con una nota igual o superior a 5 puntos.

La participación en el blog consistió en la realización de comentarios a las entradas que fue publicando el profesor. El texto de los comentarios escritos se publicaba en el blog, mientras que los comentarios orales se hacían llegar grabándolos en vídeo, subiéndolo a YouTube, e indicando en el comentario a la entrada del blog el enlace para acceder al vídeo. El profesor publicó cinco entradas a lo largo de la asignatura. Tres de ellas requirieron un comentario por escrito y las otras dos un comentario oral. Los alumnos tuvieron siempre una semana de plazo para realizar sus comentarios a las distintas entradas. Cada estudiante podía realizar un solo comentario en cada entrada.

Los comentarios que cumplieron los criterios de calidad establecidos por el profesor (ver apartado 3.2) fueron premiados con 0,1 puntos, que se añadían a la nota final de la asignatura. En caso de que los alumnos suspendieran el examen, la nota obtenida con la participación en el blog se guardaba para las convocatorias de septiembre y diciembre.

Al explicar el sistema de evaluación, se advirtió a los estudiantes que los comentarios irrespetuosos o de muy baja calidad podían no solo no ser premiados, sino bajar 0,1 en la nota final de la asignatura.

\subsection{Los criterios de evaluación de los comentarios}

Los criterios de evaluación de los comentarios a las entradas del blog fueron definidos por el profesor y comunicados al alumnado el día de la presentación de la asignatura y su guía docente. Estos criterios fueron los siguientes:

\subsubsection{Criterios de evaluación para comentarios expresados por escrito}

- Calidad de las ideas (buenas ideas por ser útiles e invitar a los lectores a comportarse de un modo más inteligente y adecuado en la sociedad, por ser originales y ofrecer nuevas perspectivas del tema que se está discutiendo, por estar bien justificadas y por ser éticamente adecuadas).

- Orden de las ideas (existe un hilo conductor, el orden de las ideas es adecuado, no se repite la misma idea al principio y al final del texto, se nota que las ideas se han pensado y ordenado antes de redactar).

- Calidad de la expresión escrita (bien escrito, fácil de leer y entender, sin faltas de ortografía ni abreviaturas como "xq", impropias del lenguaje académico).

- Cantidad de información (que se aproxime a las 400 palabras de límite, sin pasarse).

3.2.2. Criterios de evaluación para comentarios orales grabados en vídeo 
Uso de un blog docente para el desarrollo del pensamiento crítico y la capacidad de expresión oral y escrita en ciencias del deporte.

- Calidad de las ideas (buenas ideas por ser útiles e invitar a los espectadores a comportarse de un modo más inteligente y adecuado en la sociedad, por ser originales y ofrecer nuevas perspectivas del tema que se está discutiendo, por estar bien justificadas y por ser éticamente adecuadas).

- Orden de las ideas (existe un hilo conductor, el orden de las ideas es adecuado, no se repite la misma idea al principio y al final del vídeo, se nota que las ideas se han pensado y ordenado antes de la exposición oral).

- Calidad de la expresión oral (uso adecuado del lenguaje oral y corporal, discurso fácil de seguir y que se entienda bien, evitar vocabulario que pueda resultar irrespetuoso, no leer ningún papel).

- El vídeo debe ser de una duración superior a 1 minuto e inferior a 2 minutos.

\subsection{Procedimiento de evaluación}

Para evaluar los comentarios se utilizó un sistema de coevaluación, en el que los evaluadores de los comentarios eran alumnos que se prestaban voluntariamente a realizar esta labor. Los alumnos interesados en ser evaluadores debían escribir un e-mail al profesor y los cinco primeros que lo hicieron fueron elegidos como evaluadores. Cada uno de los cinco evaluadores debía encargarse de realizar su función en una de las cinco entradas del blog.

Estos evaluadores eran anónimos para el resto de compañeros, con el objetivo de que pudieran sentirse libres para ejercer su función, sin temor a problemas de relación con los compañeros por no premiar algún comentario.

Cuando se publicaba la entrada que debían evaluar, el evaluador recibía un e-mail por parte del profesor, que contenía las siguientes indicaciones respecto al procedimiento de evaluación:

- Leer los comentarios de los compañeros. En un archivo Word, redactar un informe con los apartados que se indican a continuación:

1) Premiados (indicar los apellidos y el nombre de todos los premiados, primero los apellidos, y ordenados alfabéticamente. Indicar también cuál es el mejor comentario a juicio del evaluador).

2) No premiados (indicar apellidos y nombre de los no premiados, ordenados alfabéticamente, y las razones para no premiar cada uno de los comentarios).

3) Resumen de las ideas de los compañeros (anotar y ordenar las ideas principales que van apareciendo, en unos 4-5 párrafos de 2-3 líneas aproximadamente). Una vez terminado este proceso, el evaluador enviará por e-mail el informe al profesor. El plazo para la entrega de este documento es de una semana a partir de la fecha en la que acaba el plazo para realizar comentarios. El profesor asignará 0,1 al evaluador y 0,1 a cada alumno cuyo comentario haya sido premiado. El profesor también presentará en clase el resumen de las ideas elaborado por el evaluador del blog.

(cc) EY-NC-ND 2017, Universitat Politècnica de València 
- El evaluador debe tratar de premiar bastantes comentarios para que los alumnos se animen a participar en futuras entradas. No hay ningún problema si se premian todos los comentarios. Eso sí, se debe evitar que un comentario que sea claramente de baja calidad según los criterios de evaluación resulte premiado. Es importante que el alumno perciba que no vale cualquier cosa.

\subsection{Las entradas publicadas}

La entradas publicadas fueron las siguientes:

\subsubsection{Entrada 1: Carreras de larga duración ... ¿Vida o muerte? (Comentario escrito).}

Una vez leído el artículo cuyo enlace se muestra a continuación, ¿qué contestaríais al autor? http://www.elmundo.es/blogs/elmundo/guantanamo/2012/10/01/desperdiciar-la-vida.html

Este enlace vinculaba a un artículo de Salvador Sostres publicado en el diario El Mundo, en el que el autor realiza una dura crítica a los participantes y organizadores de carreras de larga duración.

\subsubsection{Entrada 2: Éxito profesional (Comentario oral).}

En un futuro cercano acabaréis vuestro grado y buscaréis trabajo. Por eso me gustaría preguntaros lo siguiente: ¿Qué significa para vosotros el éxito profesional? ¿En qué circunstancias os consideraríais con éxito profesional?

\subsubsection{Entrada 3: La gestión de la fama (Comentario escrito).}

Una vez visto el vídeo de Valentino Rossi, cuyo enlace se muestra a continuación, ¿qué opinión queréis manifestar?

https://www.youtube.com/watch?v=w5YkwaJFlvw

Este enlace vinculaba a un vídeo de YouTube en el que Valentino Rossi parece dar una patada a una aficionada cuando trata de avanzar en moto entre la multitud.

3.4.4. Deporte y medio ambiente (Comentario escrito).

¿Qué puedes hacer en un futuro, como profesional de las ciencias del deporte, para promocionar el respeto al medio ambiente?

\subsubsection{Fútbol y evasión fiscal (Comentario oral).}

¿Crees que verdaderamente existe evasión fiscal por parte de futbolistas famosos? ¿Qué opinas de esta situación? Presenta tu comentario oral sin mencionar a ningún futbolista famoso en concreto.

\subsection{Presentación del resumen y concesión del premio simbólico al mejor comentario}

Una ver recibido el informe del evaluador, el profesor presentaba el resumen de las ideas procedentes de los comentarios de los alumnos. Posteriormente, el profesor aportaba alguna reflexión u opinión personal sobre el tema de la entrada. Finalmente, daba de nuevo la voz a los estudiantes para establecer una breve discusión y llegar a conclusiones finales. 
Uso de un blog docente para el desarrollo del pensamiento crítico y la capacidad de expresión oral y escrita en ciencias del deporte.

Tras finalizar este proceso, el profesor nombraba al estudiante que, a juicio del evaluador, había realizado el mejor comentario para que se acercara y entregarle un regalo de "valor simbólico y no material", como muestra de su agradecimiento y reconocimiento ante el buen trabajo realizado. Esto regalos fueron pequeños obsequios que había recibido el profesor por su asistencia a congresos científicos. Por ejemplo, un llavero, una bolsa de congreso, una libreta, etc.

En el momento de conceder el regalo, el profesor pensaba unas palabras para darle mayor solemnidad a la entrega, con ciertas dosis de humor. Por ejemplo, "te entrego este llavero, para que lleves siempre contigo la llave del conocimiento, que tantas puertas te abrirá en la vida". Tras la entrega, toda la clase aplaudía al alumno premiado.

\section{Resultados}

La primera entrada recibió el comentario por escrito de 33 estudiantes, la segunda entrada recibió el comentario oral de 12 estudiantes, la tercera entrada recibió el comentario escrito de 35 estudiantes, la cuarta entrada recibió el comentario escrito de 29 estudiantes, la quinta entrada recibió el comentario oral de 8 estudiantes.

Todos los evaluadores cumplieron a la perfección con su función. Además, la inmensa mayoría de los comentarios fueron premiados por cumplir con los criterios de evaluación establecidos.

Una vez finalizada la asignatura, el profesor envío al alumnado un formulario de Google para conocer sus opiniones sobre el blog. Este formulario fue contestado por 24 estudiantes. De ellos, 16 estudiantes habían realizado algún comentario en el blog, mientras que 8 no habían realizado ningún comentario.

Los alumnos que habían participado en el blog manifestaron, en sus respuestas a una pregunta abierta, haberlo hecho por los siguientes motivos:

- Los temas de las entradas les parecían interesantes.

- Podían subir nota.

- La participación en el blog les servía para mejorar su capacidad de expresión oral y escrita y la capacidad de pensamiento crítico, habilidades necesarias en cualquier ámbito profesional.

- La participación en el blog les servía para hacerles pensar y reflexionar sobre temas de actualidad.

- Les parecía una actividad entretenida.

- Se discutían y ofrecían posibles soluciones a los problemas relacionados con los temas de la entrada.

Los alumnos que no participaron en la entrada manifestaron, en sus respuestas a una pregunta abierta, no haberlo hecho por los siguientes motivos:

- Falta de tiempo, especialmente en el caso de alumnos que compaginaban trabajo y estudios. Este fue el motivo expresado con mayor frecuencia.

- Por pensar que no podría hacer un comentario de suficiente calidad que pudiera llegar incluyo a restarles nota. Este motivo fue expresado por dos estudiantes.

(cc) EY-NC-ND 2017, Universitat Politècnica de València 
- Por problemas técnicos relacionados con la publicación de la entrada. Un alumno comentó que intentó publicar su comentario en un par de ocasiones y no quedó registrado. Esto le desanimó para participar en futuras entradas.

Posteriormente, en el cuestionario se planteaba una pregunta de respuestas cerradas, expresada en los siguientes términos:

Creo que el blog de la asignatura, como recurso docente, puede ser útil para...

En la Tabla 1 se concreta la frecuencia (n) y el porcentaje (\%) de alumnos que señalaron cada respuesta.

Tabla 1. Utilidades del blog como recurso docente según los alumos. Frecuencia y porcentaje de alumnos que señaló cada una de las posibles respuestas

\begin{tabular}{lcc}
\hline Posibles respuestas & n & $\%$ \\
\hline Favorecer la capacidad de expresión oral y escrita & 22 & 91,6 \\
Favorecer el pensamiento crítico & 21 & 87,5 \\
Mejorar la capacidad de exponer, defender y debatir ideas & 18 & 75 \\
Fomentar el interés por estar informado y adquirir nuevos conocimientos & 15 & 62,5 \\
Incrementar el interés por la asignatura & 13 & 54,1 \\
Fomentar la ética profesional & 12 & 50 \\
Favorecer nuestra conciencia social/medioambiental y nuestro compromiso & 12 & 50 \\
con una sociedad más justa & & 0 \\
Creo que no vale para nada & 0 & 0 \\
\hline
\end{tabular}

Nota: El cuestionario fue cumplimentado por 24 estudiantes.

Finalmente, el cuestionario acababa con la siguiente pregunta de respuesta abierta:

¿Crees que la participación en el blog puede ser útil para algo más? Indica para qué.

Los estudiantes comentaron que el blog también podía ser de utilidad para comprender mejor algunas partes de la asignatura, para romper con la rutina, captar su atención y hacer más entretenida la asignatura. Asimismo, destacaron que el blog generaba cierta competitividad por hacer los mejores comentarios que les resultaba atractiva.

\section{Conclusiones}

El nivel de participación en el blog fue aceptable, si tenemos en cuenta que se trató de un trabajo voluntario. Probablemente, los alumnos percibieron el blog como un recurso 
Uso de un blog docente para el desarrollo del pensamiento crítico y la capacidad de expresión oral y escrita en ciencias del deporte.

docente útil y formativo porque el trabajo era voluntario y sólo aquellos con verdadero interés participaron. Además, esta experiencia docente fue muy satisfactoria para el profesor de la asignatura. No se pone en duda que la exigencia, por parte del profesorado, de trabajos obligatorios puede ser interesante para el aprendizaje del alumno. Sin embargo, en este tipo de trabajos existe el riesgo de que muchos estudiantes no muestren el nivel de implicación y rigor esperado por el profesor. Esto puede acabar desencadenando experiencias docentes poco motivantes, que a la larga pueden acabar mermando la vocación e implicación del profesor en tareas docentes.

La participación en comentarios orales fue claramente más baja que la participación por medio de comentarios escritos. La razón de esta diferencia no quedó clara con los motivos expresados por los estudiantes para no realizar comentarios en el blog. Quizá se realizaron menos comentarios orales porque el proceso de elaboración podría ser más costoso, o porque les diera vergüenza que su vídeo pudiera ser visto por otros compañeros de clase. No obstante, en futuras experiencias se debería preguntar directamente acerca de esta cuestión.

La percepción del profesor de esta asignatura fue que, tanto los estudiantes que realizaron comentarios como los evaluadores, participaron en el blog con rigor y responsabilidad. El profesor también percibió que el uso del blog sirvió para conseguir los objetivos educativos propuestos. Las respuestas de los estudiantes al formulario de Google refuerzan igualmente la idea de que el blog fue un recurso educativo útil para el desarrollo del pensamiento crítico y la capacidad de expresión oral y escrita. En este sentido, otras experiencias con edublogs han sugerido que los blogs son recursos educativos interesantes para la formación de los estudiantes (Molina Alventosa, Valenciano Valcárcel y Valencia Peris, 2015; Molina Alventosa, Valencia Peris y Suárez Guerrero, 2016).

Por último, la concesión de un premio simbólico por parte del profesor al mejor comentario según el evaluador, pareció fomentar cierta competitividad que motivaba a los alumnos a demostrar al profesor y al resto de compañeros que ellos también eran capaces de realizar comentarios acertados e inteligentes. Esta situación, bien canalizada, podría tener cierto valor educativo, si la competitividad nos lleva a tratar de sorprender con comentarios inteligentes a unos compañeros y a un profesor por los que sentimos respeto intelectual. Sin embargo, una competitividad irrespetuosa, que inhibiera la participación de los alumnos que se perciben menos competentes, o generadora de envidias y recelos, acabaría siendo negativa para el proceso de enseñanza-aprendizaje.

\section{Referencias}

BOISVERT, J. (2004). La formación del pensamiento crítico. Teoría y práctica. México. Fondo de cultura económica.

GUZMAN SILVA, S. y Sánchez Escobedo, P, (2006). "Efectos de un programa de capacitación de profesores en el desarrollo de habilidades de pensamiento crítico en estudiantes universitarios en el Sureste de México" en Revista Electrónica de Investigación Educativa (REDIE), vol. 2, no 2, [http://redie.uabc.mx/vol8no2/contenido-guzman.html]

(c) EY-NC-ND 2017, Universitat Politècnica de València 
MOLINA ALVENTOSA, J. P., VALENCIANO VALCÁRCEL, J. y VALENCIA PERIS, A. (2015). "Los blogs como entornos virtuales de enseñanza aprendizaje en Educación Superior" en Revista Complutense de Educación, vol. 26 (especial TIC en Educación), pp. 15-31.

MOLINA ALVENTOSA, J. P., VALENCIA PERIS, A. y SUÁREZ, C. (2016). "Percepción de los estudiantes de una experiencia de uso didáctico de blog docente en Educación Superior" en Educación XX1, vol. 19, n 1, pp. 91-113.

PEÑA GONZÁLEZ, F. J. (2011). "Leer y escribir. Prácticas necesarias en la universidad" en Educere, vol. 15, nº 52, pp. 711-719.

ROJAS VITERI, J.C., PÉREZ NARVÁEZ, H.O. y ÁLVAREZ ZURITA, A.M. (2016). “El pensamiento crítico en la educación” en Revista Publicando, vol. 3, n 9, pp. 110-118.

RUIZ MUÑOZ, M. J. (2012). "El desarrollo de competencias orales y escritas en el marco del Espacio Europeo de Educación Superior (EEES). Reflexiones, propuestas y experiencias en el Grado en Publicidad y Relaciones Públicas" en Revista de Comunicación Vivat Academia, año XIV, $\mathrm{n}^{\circ}$ Especial, pp. 133-144. 